\title{
An Extended Kalman Filter with a Computed Mean Square Error Bound
}

\author{
G. Hexner, and H. Weiss*
}

\begin{abstract}
The paper proposes a new recursive filter for non-linear systems that inherently computes a valid bound on the mean square estimation error. The proposed filter, bound based extended Kalman, (BEKF) is in the form of an extended Kalman filter. The main difference of the proposed filter from the conventional extended Kalman filter is in the use of a computed mean square error bound matrix, to calculate the filter gain, and to serve as bound on the actual mean square error. The paper shows that when the system is linear the proposed filtering algorithm reduces to the conventional Kalman filter. The theory presented in the paper is applicable to a wide class of systems, but if the system is polynomial, then the recently developed theory of positive polynomials considerably simplifies the filter's implementation.
\end{abstract}

\section{INTRODUCTION}

The purpose of this paper is to propose a new form of extended Kalman filter (BEKF) with a computed bound on the mean squared error matrix, used to calculate the filter gain and to serve as a bound on the actual estimate mean square error. The theory presented is general, subject only to some asymptotic growth and continuity constraints, but implementation is very much simplified if the underlying system consists of rational polynomials. In this case the recently introduced theory of positive polynomials [1], [2], [3], and the software SOSTOOLS [4] provides the tools for efficient implmentation of the filter. SOSTOOLS translates the problem to a semi-definite program, which is readily solved by SeDuMi [5]. This set of software makes possible the numerical calculation of the bounds necessary in this paper routine.

Previous attempts at extended Kalman filtering for polynomial based systems inevitably faced the closure problem [6], [7]. The closure problem refers to the fact that to calculate the $n$th moment of a distribution, the value of the $n+1$ and possibly higher order moments are required. A popular method has been to assume that moments of higher order are related to lower order moments as if the underlying probability density were Gaussian, [7].

One approach to non-linear estimation was presented in [8] based on a special type of discretization of the exact equations of nonlinear filtering. A different approach to estimation for non-linear systems was proposed for cone bounded non-linearities in [9], [10], [11]. The special feature of these papers, compared to the many publications that deal with estimation for non-linear systems, is the derivation of an analytic bound on the performance of the estimator, without requiring any sort of truncation approximation. The present

\footnotetext{
* The authors are with RAFAEL, Advanced Defense Systems, \{georgeh, haimw\}@rafael.co.il
}

paper proposes to derive an analytic bound on the mean square estimation error using an alternative approach.

For proper operation of any Kalman filter it is essential that the calculated filter mean square error track the actual filter mean square error reasonably well. The reason for this is that the filter mean square error defines the filter gain. Too small value of the calculated mean square error implies that the Kalman gain is too low and the observations are insufficiently weighted in updating the filter estimate. The formalization of the concept is called consistency, [12]. For a filter to be consistent two conditions have to be fulfilled:

1) Have mean zero (i. e. the estimates are unbiased)

2) Have covariance matrix as calculated by the filter.

The exact methods for testing and consequences of filter consistency are discussed further in [12]. The proposed filter (BEKF) does not ensure that the estimate error is zero mean, but does ensure that the filter mean square error calculated by the algorithm dominates the actual mean square error. Thus it cannot be claimed that BEKF is consistent; however, it does ensure a reasonable value for the filter gain.

An important step in the development of an extended Kalman filter is "tuning". This consists of adjusting (usually increasing) by trial and error the intensity of the process noise and possibly the observation noise so that the filter calculated mean square error is in some agreement with the actual mean square error. The contribution of the present paper is the development of an algorithm that ensures that the calculated mean square estimation error is larger or equal to the actual estimation error. In particular the new filter precludes the possibility filter divergence.

\section{Problem Statement}

Let $\boldsymbol{x}$ be an $N$ dimensional diffusion,

$$
\mathrm{d} \boldsymbol{x}=\boldsymbol{f}(\boldsymbol{x}) \mathrm{d} t+\boldsymbol{g}(\boldsymbol{x}) \mathrm{d} \boldsymbol{w}
$$

where $\boldsymbol{w}$ is a standard vector Weiner process. The variable $\boldsymbol{y}$ is observed at time instances $T_{k}, k=0,1, \ldots$

$$
\boldsymbol{y}\left(T_{k}\right)=\boldsymbol{H} \boldsymbol{x}\left(T_{k}\right)+\boldsymbol{v}_{k}
$$

where $\boldsymbol{v}_{k}$ is a sequence of zero mean independent variables with covariance matrix $\boldsymbol{R}$. Also the initial mean squared error matrix $\boldsymbol{\Sigma}(0)$ of the initial value of the state vector $\boldsymbol{x}(0)$ is assumed known. The following is necessary for the development of the bound:

Assumption 1: The function $\boldsymbol{g}(\boldsymbol{x})$ is continuous and the function $f(x)$ is continuously differentiable. 
Assumption 2: For any symmetric matrix $\boldsymbol{P}$ there exists a symmetric matrix $Q$ and a constant $q$ such that

$$
\left(\frac{\partial \boldsymbol{f}(\boldsymbol{x})}{\partial \boldsymbol{x}}\right)^{\prime} \boldsymbol{P}+\boldsymbol{P}\left(\frac{\partial \boldsymbol{f}(\boldsymbol{x})}{\partial \boldsymbol{x}}\right) \preceq \boldsymbol{Q}
$$

and

$$
\operatorname{Tr}\left\{\boldsymbol{g}^{\prime}(\boldsymbol{x}) \boldsymbol{P} \boldsymbol{g}(\boldsymbol{x})\right\} \leq q
$$

Note that there are no sign definite constraints on $\boldsymbol{P}$ or $Q$ in (3). Assumption 2 essentially limits the growth of $\|\boldsymbol{f}(\boldsymbol{x})\|$ to be at most linear in $\boldsymbol{x}$, for large $\|\boldsymbol{x}\|$. The form on the left hand side of (3) occurs in the study of contracting systems [13]. When $\boldsymbol{P} \succ \mathbf{0}$ the form measures the rate that two solutions of (1), with close initial conditions diverge from each other. In [14] it is shown that if $\boldsymbol{P} \succ \mathbf{0}$ and if $Q \prec \mathbf{0}$ in (3) in the whole space and (4) is valid then (1) is incrementally stochastically stable.

The aim of the paper is to develop an algorithm for calculating an estimate, $\hat{x}$ of the state of (1), based on the observations (2) and a guaranteed bound ${ }^{1}$ for the expected value of the mean square error of the estimate.

\section{A. Some Examples of Systems Satisfying Assumption 2}

The simplest system satisfying Assumption 2 is

$$
\mathrm{d} \boldsymbol{x}=\boldsymbol{A}(\boldsymbol{x}) \boldsymbol{x} \mathrm{d} t+\boldsymbol{g}(\boldsymbol{x}) \mathrm{d} \boldsymbol{w}
$$

where $\boldsymbol{A}(\boldsymbol{x})$ and $\boldsymbol{g}(\boldsymbol{x})$ satisfy

$$
\|\boldsymbol{A}(\boldsymbol{x})\|+\left\|\frac{\partial \boldsymbol{A}(\boldsymbol{x})}{\partial \boldsymbol{x}}\right\| \leq M_{\boldsymbol{A}} ; \quad\|\boldsymbol{g}(\boldsymbol{x})\| \leq M_{\boldsymbol{g}}
$$

for some constants $M_{\boldsymbol{A}}$, and $M_{\boldsymbol{g}}$.

A system which contradicts assumption 2 is

$$
\mathrm{d} x=\left(1+x^{2}\right) d t+d w
$$

Note that the system (7) has a finite escape time.

\section{TIME UPDATE}

Between observations the state estimate, $\tilde{\boldsymbol{x}}$ evolves as in the extended Kalman filter, according to

$$
\mathrm{d} \tilde{\boldsymbol{x}}=\boldsymbol{f}(\tilde{\boldsymbol{x}}) \mathrm{d} t
$$

The initial condition for $\tilde{\boldsymbol{x}}$ after the $k$ th data processing step is $\hat{\boldsymbol{x}}_{k}$. Then the estimate error, $\tilde{\boldsymbol{e}}=\tilde{\boldsymbol{x}}-\boldsymbol{x}$ evolves as

$$
\mathrm{d} \tilde{\boldsymbol{e}}=\boldsymbol{F}(\tilde{\boldsymbol{e}}, \tilde{\boldsymbol{x}}) \mathrm{d} t+\boldsymbol{G}(\tilde{\boldsymbol{e}}, \tilde{\boldsymbol{x}}) \mathrm{d} \boldsymbol{w}
$$

where

$$
\begin{gathered}
F(\tilde{e}, \tilde{x})=f(\tilde{x})-f(\tilde{x}-\tilde{e}) \\
G(\tilde{e}, \tilde{x})=-g(\tilde{x}-\tilde{e})
\end{gathered}
$$

Note that $\tilde{\boldsymbol{x}}$ is computed according to (8), so that it is a known function.

An important contribution of the paper is the calculation of $\tilde{\Sigma}$ such that

$$
\mathbf{E}\left\{\tilde{\boldsymbol{e}} \tilde{\boldsymbol{e}}^{\prime}\right\}=\boldsymbol{\Sigma} \preceq \tilde{\boldsymbol{\Sigma}}
$$

${ }^{1}$ Note that the emphasis here is the development of a bound. No attempt is made here to develop tightest possible bound is a bound on the mean square error, $\mathbf{E}\left\{\tilde{\boldsymbol{e}} \tilde{\boldsymbol{e}}^{\prime}\right\}=\boldsymbol{\Sigma}$. This is accomplished by calculating bounds for

$$
\operatorname{Tr}\{\boldsymbol{P} \dot{\boldsymbol{\Sigma}}\}
$$

Using the Ito calculus [7],

$$
\begin{aligned}
\mathrm{d}\left[\tilde{\boldsymbol{e}}^{\prime} \boldsymbol{P} \tilde{\boldsymbol{e}}\right]= & \mathrm{d} \tilde{\boldsymbol{e}}^{\prime} \boldsymbol{P} \tilde{\boldsymbol{e}}+\tilde{\boldsymbol{e}}^{\prime} \boldsymbol{P} \mathrm{d} \tilde{\boldsymbol{e}}+\mathrm{d} \tilde{\boldsymbol{e}}^{\prime} \boldsymbol{P} \mathrm{d} \tilde{\boldsymbol{e}} \\
= & {\left[\boldsymbol{F}^{\prime}(\tilde{\boldsymbol{e}}, \tilde{\boldsymbol{x}}) \boldsymbol{P} \tilde{\boldsymbol{e}}+\tilde{\boldsymbol{e}}^{\prime} \boldsymbol{P} \boldsymbol{F}(\tilde{\boldsymbol{e}}, \tilde{\boldsymbol{x}})\right] } \\
& +\operatorname{Tr}\left\{\boldsymbol{G}^{\prime}(\tilde{\boldsymbol{e}}, \tilde{\boldsymbol{x}}) \boldsymbol{P} \boldsymbol{G}(\tilde{\boldsymbol{e}}, \tilde{\boldsymbol{x}})\right\} \mathrm{d} t \\
& +\mathrm{d} \boldsymbol{w}^{\prime} \boldsymbol{G}^{\prime}(\tilde{\boldsymbol{e}}, \tilde{\boldsymbol{x}}) \boldsymbol{P} \tilde{\boldsymbol{e}}+\tilde{\boldsymbol{e}}^{\prime} \boldsymbol{P} \boldsymbol{G}(\tilde{\boldsymbol{e}}, \tilde{\boldsymbol{x}}) \mathrm{d} \boldsymbol{w}
\end{aligned}
$$

Taking expectation in (15), and simplifying

$$
\begin{aligned}
\operatorname{Tr}\{\boldsymbol{P} \dot{\boldsymbol{\Sigma}}\}= & \mathbf{E}\left\{\boldsymbol{F}^{\prime}(\tilde{\boldsymbol{e}}, \tilde{\boldsymbol{x}}) \boldsymbol{P} \tilde{\boldsymbol{e}}+\tilde{\boldsymbol{e}}^{\prime} \boldsymbol{P F}(\tilde{\boldsymbol{e}}, \tilde{\boldsymbol{x}})\right. \\
& \left.+\operatorname{Tr}\left\{\boldsymbol{G}^{\prime}(\tilde{\boldsymbol{e}}, \tilde{\boldsymbol{x}}) \boldsymbol{P} \boldsymbol{G}(\tilde{\boldsymbol{e}}, \tilde{\boldsymbol{x}})\right\}\right\}
\end{aligned}
$$

Using the mean value theorem,

$$
F(\tilde{e}, \tilde{x})=f(\tilde{x})-f(\tilde{x}-\tilde{e})=\frac{\partial f(c)}{\partial x} \tilde{e}
$$

where $\boldsymbol{c}$ is a point on the line connecting $\tilde{\boldsymbol{x}}$ and $\tilde{\boldsymbol{x}}-\tilde{\boldsymbol{e}}$. Substituting (17) in (16),

$$
\begin{array}{r}
\operatorname{Tr}\{\boldsymbol{P} \dot{\Sigma}\}=\mathbf{E}\left\{\tilde{\boldsymbol{e}}^{\prime}\left(\frac{\partial \boldsymbol{f}}{\partial \boldsymbol{x}}\right)^{\prime} \boldsymbol{P} \tilde{\boldsymbol{e}}+\tilde{\boldsymbol{e}}^{\prime} \boldsymbol{P}\left(\frac{\partial \boldsymbol{f}}{\partial \boldsymbol{x}}\right) \tilde{\boldsymbol{e}}\right\} \\
+\operatorname{Tr} \mathbf{E}\left\{\boldsymbol{G}^{\prime}(\tilde{\boldsymbol{e}}, \tilde{\boldsymbol{x}}) \boldsymbol{P G}(\tilde{\boldsymbol{e}}, \tilde{\boldsymbol{x}})\right\}
\end{array}
$$

taking expectations and using (3) yields the inequality

$$
\operatorname{Tr}\{\boldsymbol{P} \dot{\boldsymbol{\Sigma}}\} \leq \operatorname{Tr}\{\boldsymbol{Q} \boldsymbol{\Sigma}\}+q
$$

Therefore assumptions 1 and 2 ensure that for every $\boldsymbol{P}$ there exist a $Q$ and $q$ satisfying (19).

In the next subsection a bound $\dot{\bar{\Sigma}}$ for $\dot{\Sigma}$ is calculated based on the repeated use of (19), using a set of $\boldsymbol{P}_{i}, \boldsymbol{Q}_{i}$, and $q_{i}$. Then to each value of $\boldsymbol{\Sigma}$ the algorithm to be presented in the next subsection calculates a bound $\dot{\bar{\Sigma}}$ for $\dot{\Sigma}$. That is, the algorithm defines a function, $\dot{\bar{\Sigma}}(\boldsymbol{\Sigma}, t)$. In the succeeding subsection, based on this function, a differential equation for the bound $\tilde{\boldsymbol{\Sigma}}$ for $\boldsymbol{\Sigma}$ is derived.

\section{A. The Bound for $\dot{\boldsymbol{\Sigma}}$}

Given a value for the mean squared error matrix, $\boldsymbol{\Sigma}$ the procedure, to be presented in this section, calculates a bound for its derivative. Here, the bound for the derivative is denoted as $\dot{\bar{\Sigma}}$. Although, the bound depends on $\boldsymbol{\Sigma}$ and $t$, and hence is a function of $\boldsymbol{\Sigma}$ and $t$, in the present subsection, this dependence is suppressed. The calculation of the bound is carried out in two steps:

1) A bounded set $\mathcal{S}$ containing $\dot{\boldsymbol{\Sigma}}$ is calculated.

2) A single $\dot{\bar{\Sigma}}$ such that

$$
\dot{\Sigma} \preceq \dot{\bar{\Sigma}}, \forall \dot{\Sigma} \in \mathcal{S}
$$

is calculated. 
1) Calculation of $\mathcal{S}$ : The set $\mathcal{S}$ is defined as

$$
\mathcal{S}(\boldsymbol{\Sigma})=\left\{\dot{\boldsymbol{\Sigma}} \mid \operatorname{Tr}\left\{\boldsymbol{P}_{i} \dot{\boldsymbol{\Sigma}}\right\} \leq \operatorname{Tr}\left\{\boldsymbol{Q}_{i} \boldsymbol{\Sigma}\right\}+q_{i}, \forall i\right\}
$$

where the set of $N \times N$ symmetric matrices $\boldsymbol{P}_{i}$ satisfy the following condition: for any symmetric $N \times N$, non-zero $\boldsymbol{Z}$ there exists an $i$ such that

$$
\operatorname{Tr}\left\{\boldsymbol{P}_{i} \boldsymbol{Z}\right\}>0
$$

This condition ensures that if $\dot{\boldsymbol{\Sigma}}$ becomes unbounded, at least one of the inequalities in (21) is violated. Note that $\mathcal{S}$ is a polytope. The construction of a set of $\boldsymbol{P}_{i}$ satisfying (22) is presented in appendix B, and the algorithm for calculating the corresponding $\boldsymbol{Q}_{i}$, and $q_{i}$ is presented in section III-C.

2) Calculation of $\dot{\bar{\Sigma}}$ : This section relies on generalized inequalities with respect various proper cones, see [15]. The generalized inequality with respect to the cone $\mathcal{A}$ is denoted as $\preceq_{\mathcal{A}}$. Ideally a matrix $\dot{\bar{\Sigma}}$ is sought such that

$$
\dot{\boldsymbol{\Sigma}} \preceq_{\mathcal{M}_{+}} \dot{\bar{\Sigma}}, \forall \dot{\boldsymbol{\Sigma}} \in \mathcal{S}
$$

where $\mathcal{M}_{+}$is the cone of positive semi-definite matrices. (This is the same as (20), while explicitly indicating the cone for the generalized inequality.) This is an infinite dimensional problem. Let $\mathcal{F}_{+}$denote the cone with a finite set of generators, $\boldsymbol{U}_{i} \succeq_{\mathcal{M}_{+}} \mathbf{0}$. Then any $\dot{\bar{\Sigma}}$ that satisfies

$$
\dot{\boldsymbol{\Sigma}} \preceq_{\mathcal{F}_{+}} \dot{\bar{\Sigma}}, \forall \dot{\boldsymbol{\Sigma}} \in \mathcal{S}
$$

also satisfies (23), since $\mathcal{F}_{+} \subseteq \mathcal{M}_{+}$. Any set of $\boldsymbol{U}_{i} \succeq_{\mathcal{M}_{+}} \mathbf{0}$ yields a valid bound, $\dot{\bar{\Sigma}}$, but the bound becomes tighter as $\mathcal{F}_{+}$approaches $\mathcal{M}_{+}$.

From the geometry of $\mathcal{M}_{+}$for maximal $\mathcal{F}_{+}$, the $\boldsymbol{U}_{i}$ should be on the boundary of $\mathcal{M}_{+}$, that is, the $\boldsymbol{U}_{i}$ should be rank one matrices. For the example for second order systems a reasonable choice is the four positive semi-definite rank one matrices

$$
\left[\begin{array}{ll}
1 & 0 \\
0 & 0
\end{array}\right],\left[\begin{array}{ll}
0 & 0 \\
0 & 1
\end{array}\right],\left[\begin{array}{ll}
1 & 1 \\
1 & 1
\end{array}\right],\left[\begin{array}{cc}
1 & -1 \\
-1 & 1
\end{array}\right]
$$

These matrices served as the $\boldsymbol{U}_{i}$ for the example in section VI. Note that there exist positive definite matrices that cannot be expressed as a positive combination of these $\boldsymbol{U}_{i}$, one example of such a positive definite matrix is

$$
\left[\begin{array}{cc}
1+2 \epsilon & 1 \\
1 & \frac{1+\epsilon}{1+2 \epsilon}
\end{array}\right]
$$

for any $\epsilon>0$. That is $\mathcal{F}_{+} \subset \mathcal{M}_{+}$.

Let $\boldsymbol{T}_{i}$ be the finite set of generators for the dual cone $\mathcal{F}_{+}^{*}$. The calculation of the $\boldsymbol{T}_{i}$, from a given set of $\boldsymbol{U}_{i}$ is discussed in appendix A. From the properties of dual cones, [15] since $\mathcal{F}_{+} \subseteq \mathcal{M}_{+}$then $\mathcal{F}_{+}^{*} \supseteq \mathcal{M}_{+}^{*}=\mathcal{M}_{+}$. Therefore, for any $\boldsymbol{T} \succeq \mathbf{0}$ there exist $\lambda_{i} \geq 0$ such that

$$
\boldsymbol{T}=\sum_{i} \lambda_{i} \boldsymbol{T}_{i}
$$

Define $t_{i}$

$$
t_{i}=\max _{\dot{\boldsymbol{\Sigma}} \in \mathcal{S}(\boldsymbol{\Sigma})} \operatorname{Tr}\left\{\boldsymbol{T}_{i} \dot{\boldsymbol{\Sigma}}\right\}
$$

Since the set $\mathcal{S}$ is bounded, the $t_{i}$ are finite. Define $\dot{\bar{\Sigma}}$

$$
\dot{\bar{\Sigma}}=\sum_{i} s_{i} \boldsymbol{T}_{i}
$$

for some $s_{i}$ yet to be specified, and define the matrix $\boldsymbol{L}$ with entries $\boldsymbol{L}_{i j}$,

$$
\boldsymbol{L}_{i j}=\operatorname{Tr}\left\{\boldsymbol{T}_{i} \boldsymbol{T}_{j}\right\}
$$

The matrix $\boldsymbol{L}$ is square with dimension equal to the number of $\boldsymbol{T}_{i}$ 's. Define the vectors $s$ and $\boldsymbol{t}$, whose entries are $s_{i}$ and $t_{j}$, respectively. Then to any $s$ that satisfies

$$
t \preceq L s
$$

there corresponds a bound $\dot{\bar{\Sigma}}$ from the assumed form (29). The calculation of $s$ is discussed at the end of the next subsection.

That the $\dot{\bar{\Sigma}}$ defined in (29) satisfies (20) follows if it can be shown that for any $\boldsymbol{T} \succeq \mathbf{0}$

$$
\operatorname{Tr}\{\boldsymbol{T}(\dot{\boldsymbol{\Sigma}}-\dot{\bar{\Sigma}})\} \leq 0
$$

Indeed, from (27) and (31),

$$
\begin{aligned}
& \operatorname{Tr}\{\boldsymbol{T}(\dot{\boldsymbol{\Sigma}}-\dot{\overline{\boldsymbol{\Sigma}}})\} \\
= & \operatorname{Tr}\left\{\sum_{i} \lambda_{i} \boldsymbol{T}_{i}(\dot{\boldsymbol{\Sigma}}-\dot{\overline{\boldsymbol{\Sigma}}})\right\} \\
\leq & \sum_{i} \lambda_{i}\left(t_{i}-\sum_{j} \boldsymbol{L}_{i j} \boldsymbol{s}_{j}\right) \\
\leq & 0
\end{aligned}
$$

\section{B. The Mean Square Bound}

For each $\boldsymbol{\Sigma}$ a bound $\dot{\bar{\Sigma}}$ for $\dot{\Sigma}$ was derived in the previous section. The purpose of the present section is to calculate $\tilde{\boldsymbol{\Sigma}}$ such that

$$
\boldsymbol{\Sigma}(t) \preceq \tilde{\boldsymbol{\Sigma}}(t), \forall t
$$

The $\dot{\bar{\Sigma}}$, calculated in the previous section depends on $\boldsymbol{\Sigma}$, and through $\tilde{\boldsymbol{x}}$ on $t$. In the present section this dependence is made explicit, and the bound is now denoted as $\dot{\bar{\Sigma}}(\boldsymbol{\Sigma}, t)$. If $\boldsymbol{\Sigma}$ were available then solving the differential equation

$$
\dot{\tilde{\Sigma}}=\dot{\bar{\Sigma}}(\boldsymbol{\Sigma}, t)
$$

would yield a bound $\tilde{\Sigma}$. The differential equation obtained by replacing $\Sigma$ by $\tilde{\Sigma}$ in $\dot{\bar{\Sigma}}(\Sigma, t)$, yields an implementable differential equation,

$$
\dot{\tilde{\Sigma}}=\dot{\bar{\Sigma}}(\tilde{\Sigma}, t)
$$

Given an initial condition it has a well defined solution. The following lemma is an extension of the comparison principle [16], p. 102 to matrix valued functions, and is used here to show that the solution of (39) provides a valid bound for $\boldsymbol{\Sigma}$.

Lemma 1: Consider the matrix valued differential equation (39) with initial condition

$$
\boldsymbol{\Sigma}\left(T_{i}\right) \preceq \tilde{\boldsymbol{\Sigma}}\left(T_{i}\right)
$$


Then

$$
\boldsymbol{\Sigma}(t) \preceq \tilde{\boldsymbol{\Sigma}}(t), t \geq T_{i}
$$

The proof of this lemma is in appendix C. Then, using this lemma the bound $\tilde{\boldsymbol{\Sigma}}$ on the mean square error is propagated between observation updates by solving (39). The updated value $\hat{\Sigma}$ of the bound $\tilde{\Sigma}$ after the observation processing step serves as the initial condition for $\tilde{\Sigma}$. Using a larger number of $\boldsymbol{T}$ 's than $n$ may make possible a tighter bound for $\boldsymbol{\Sigma}$. In this case there exist an infinite number of vectors $s$ that satisfy (31). One possibility is to chose $s$ that minimizes ${ }^{2}$

$$
\frac{\mathrm{d} \operatorname{Tr}\left\{\tilde{\boldsymbol{\Sigma}}^{2}\right\}}{\mathrm{d} t}=2 \operatorname{Tr}\{\tilde{\boldsymbol{\Sigma}} \dot{\bar{\Sigma}}\}=2 \sum_{i} s_{i} \operatorname{Tr}\left\{\tilde{\boldsymbol{\Sigma}} \boldsymbol{T}_{i}\right\}
$$

Minimizing (42) subject to (31) is a standard linear programing problem.

\section{The Calculation of $\boldsymbol{Q}_{i}$ and $q_{i}$}

The $\boldsymbol{Q}_{i}$ and $q_{i}$, required to calculate the bound $\dot{\bar{\Sigma}}$ are determined next. The calculation is made very much easier if (1) is composed of polynomials or polynomial fractions. In this case the theory of sum of squares polynomials facilitates the calculations, at a cost of being slightly conservative. For each of the $\boldsymbol{P}_{i}$ a $\boldsymbol{Q}_{i}$ and a $q_{i}$ needs to be calculated such that

$$
\begin{aligned}
& {[\boldsymbol{f}(\tilde{\boldsymbol{x}})-\boldsymbol{f}(\tilde{\boldsymbol{x}}-\tilde{\boldsymbol{e}})]^{\prime} \boldsymbol{P}_{i} \tilde{\boldsymbol{e}}+\tilde{\boldsymbol{e}}^{\prime} \boldsymbol{P}_{i}[\boldsymbol{f}(\tilde{\boldsymbol{x}})-\boldsymbol{f}(\tilde{\boldsymbol{x}}-\tilde{\boldsymbol{e}})]} \\
& +\operatorname{Tr}\left\{\boldsymbol{g}^{\prime}(\tilde{\boldsymbol{x}}-\tilde{\boldsymbol{e}}) \boldsymbol{P}_{i} \boldsymbol{g}(\tilde{\boldsymbol{x}}-\tilde{\boldsymbol{e}})\right\}-\tilde{\boldsymbol{e}}^{\prime} \boldsymbol{Q}_{i} \tilde{\boldsymbol{e}}-q_{i} \leq 0, \forall \tilde{\boldsymbol{e}}
\end{aligned}
$$

The left side of (43) is an expression in $\tilde{\boldsymbol{e}}$, with $\tilde{\boldsymbol{x}}$ a known parameter. Any $\boldsymbol{Q}_{i}$ and $q_{i}$ satisfying the inequality leads to a valid bound, but with different degree of conservatism. Assumption 2 ensures the existence of at least one $\boldsymbol{Q}_{i}$ and $q_{i}$ for any $\boldsymbol{P}_{i}$. Among all the possible $\boldsymbol{Q}_{i}$ and $q_{i}$ that satisfy (43), a reasonable choice for $\boldsymbol{Q}_{\boldsymbol{i}}$ and $q_{i}$ are the values that minimize

$$
\mathbf{E}\left\{\tilde{\boldsymbol{e}}^{\prime} \boldsymbol{Q}_{i} \tilde{\boldsymbol{e}}+q_{i}\right\}=\operatorname{Tr}\left\{\boldsymbol{Q}_{i} \boldsymbol{\Sigma}\right\}+q_{i}
$$

but $\boldsymbol{\Sigma}$ is not available. A alternative is to replace $\boldsymbol{\Sigma}$ with $\tilde{\boldsymbol{\Sigma}}$ and minimize

$$
\operatorname{Tr}\left\{\boldsymbol{Q}_{i} \tilde{\boldsymbol{\Sigma}}\right\}+q_{i}
$$

subject to (43). This entails doing the minimization in real time, at each integration step. When (1) is polynomial or consists of polynomial fractions, the minimization is efficiently solved using [4]. In general the calculated $\boldsymbol{Q}_{i}$ and $q_{i}$ are functions of $\tilde{\boldsymbol{x}}$ (as well as $\tilde{\boldsymbol{\Sigma}}$ ). The calculated $\boldsymbol{Q}_{i}$ and $q_{i}$ are then used in (19).

In addition to solving the optimization (45), the maximum in (28) must be calculated. This, however, is a standard linear programming problem.

\footnotetext{
${ }^{2} \operatorname{Tr}\left\{\tilde{\boldsymbol{\Sigma}}^{2}\right\}$ is equal to the sum of squares of the eigenvalues of $\tilde{\boldsymbol{\Sigma}}$. Since $\tilde{\boldsymbol{\Sigma}} \succeq \mathbf{0}$ this leads to a type of minimum mean square error matrix. Another possibility is to minimize the trace of $\tilde{\boldsymbol{\Sigma}}$. This also leads to linear program.
}

\section{The Linear Case}

As a first demonstration of the calculation of the bound, the algorithm is applied to the linear case. In this case the diffusion (1) becomes

$$
\mathrm{d} \boldsymbol{x}=\boldsymbol{A} \boldsymbol{x} \mathrm{d} t+\boldsymbol{B} \mathrm{d} \boldsymbol{w}
$$

Assumptions 1 and 2 are trivially satisfied. For the linear case, (43) becomes,

$$
\tilde{\boldsymbol{e}}^{\prime} \boldsymbol{A}^{\prime} \boldsymbol{P}_{\boldsymbol{i}} \tilde{\boldsymbol{e}}+\tilde{\boldsymbol{e}}^{\prime} \boldsymbol{P}_{\boldsymbol{i}} \boldsymbol{A} \tilde{\boldsymbol{e}}+\operatorname{Tr}\left\{\boldsymbol{B}^{\prime} \boldsymbol{P}_{\boldsymbol{i}} \boldsymbol{B}\right\}-\tilde{\boldsymbol{e}}^{\prime} \boldsymbol{Q}_{\boldsymbol{i}} \tilde{\boldsymbol{e}}-q_{i} \leq 0
$$

Minimal $\boldsymbol{Q}_{\boldsymbol{i}}$ and $q_{i}$ satisfying (47)

$$
Q_{i}=A^{\prime} P_{i}+P_{i} A
$$

and

$$
q_{i}=\operatorname{Tr}\left\{\boldsymbol{B}^{\prime} \boldsymbol{P}_{i} \boldsymbol{B}\right\}
$$

Substituting these into (19), and rearranging,

$$
\operatorname{Tr}\left\{\boldsymbol{P}_{\boldsymbol{i}}\left(\dot{\boldsymbol{\Sigma}}-\boldsymbol{\Sigma} \boldsymbol{A}^{\prime}-\boldsymbol{A} \boldsymbol{\Sigma}-\boldsymbol{B} \boldsymbol{B}^{\prime}\right)\right\} \leq 0
$$

which, in view of (22) implies

$$
\dot{\Sigma}=\Sigma A^{\prime}+A \Sigma+B B^{\prime}
$$

Hence, for the linear case, the method of the bounds employed in the present paper in fact implies the exact equations for the propagation of the covariance.

\section{The ObSERVATION UPdate SteP}

At the start of the observation update step only a bound on the mean square error is available. Using this bound both the estimate and the mean square error bound is to be updated. The update step is restricted to be linear and of the form

$$
\hat{\boldsymbol{x}}_{k}=\tilde{\boldsymbol{x}}\left(T_{k}\right)+\boldsymbol{K}_{k}\left(\boldsymbol{y}\left(T_{k}\right)-\boldsymbol{H} \tilde{\boldsymbol{x}}\left(T_{k}\right)\right)
$$

where $\boldsymbol{K}_{k}$ is a gain matrix, which is to be determined. A reasonable criterion for the updated estimate is one that minimizes the worst case mean squared error given the bound $\tilde{\boldsymbol{\Sigma}}\left(T_{k}\right)$ on the prior mean squared error matrix $\boldsymbol{\Sigma}\left(T_{k}\right)$. The expected value of the mean squared error matrix after the update (52) is given by the Joseph form, [12].

$$
\hat{\boldsymbol{\Sigma}}_{k}=\left[\boldsymbol{I}-\boldsymbol{K}_{\boldsymbol{k}} \boldsymbol{H}\right] \boldsymbol{\Sigma}\left(T_{k}\right)\left[\boldsymbol{I}-\boldsymbol{K}_{\boldsymbol{k}} \boldsymbol{H}\right]^{\prime}+\boldsymbol{K}_{k} \boldsymbol{R} \boldsymbol{K}_{k}^{\prime}
$$

Accordingly, $\boldsymbol{K}_{k}$ is chosen as the value that achieves the mini-max in

$$
\min _{\boldsymbol{K}_{k}} \max _{\boldsymbol{\Sigma}\left(T_{k}\right) \preceq \tilde{\boldsymbol{\Sigma}}\left(T_{k}\right)} \operatorname{Tr}\left\{\hat{\boldsymbol{\Sigma}}_{k}\right\}
$$

The $\boldsymbol{\Sigma}\left(T_{k}\right)$ that achieves the inner maximum of the trace is $\tilde{\boldsymbol{\Sigma}}\left(T_{k}\right)$. The value of $\boldsymbol{K}_{k}$ that minimizes the trace of the Joseph form is given by,

$$
\boldsymbol{K}_{k}=\tilde{\boldsymbol{\Sigma}}\left(T_{k}\right) \boldsymbol{H}^{\prime}\left[\boldsymbol{R}+\boldsymbol{H} \tilde{\boldsymbol{\Sigma}}\left(T_{k}\right) \boldsymbol{H}^{\prime}\right]^{-1}
$$

This then is the well known Kalman filter gain, but with the mean squared error matrix replacing the prior covariance matrix. The updated bound on the mean squared error matrix is

$$
\hat{\boldsymbol{\Sigma}}_{k}=\left[\boldsymbol{I}-\boldsymbol{K}_{k} \boldsymbol{H}\right] \tilde{\boldsymbol{\Sigma}}\left(T_{k}\right)\left[\boldsymbol{I}-\boldsymbol{K}_{k} \boldsymbol{H}\right]^{\prime}+\boldsymbol{K}_{k} \boldsymbol{R} \boldsymbol{K}_{k}^{\prime}
$$




\section{IMPLEMENTING THE ALGORITHM}

In this section the various parts of the filtering algorithm are collected.

\section{A. Off Line Calculations}

The $\boldsymbol{P}_{i}$ and $\boldsymbol{T}_{i}$ are independent of the observations, and the filter state, therefore these matrices may be calculated off-line. The algorithm for the calculation can be found in appendices B and A. Also $\boldsymbol{L}$ is calculated at this stage (30).

\section{B. Observation Update}

The observation update very much follows the observation update of the conventional extended Kalman filter, except the prior covariance matrix is replaced by the mean square error matrix bound $\tilde{\boldsymbol{\Sigma}}$. The state is updated as in (52), the gain is calculated in (55), and the mean square error matrix is updated in (56).

\section{Time Update}

Between observations the state is propagated according to (8). The mean square error matrix is propagated by solving (39). In order to be able to carry this out the matrix function $\dot{\bar{\Sigma}}(\tilde{\boldsymbol{\Sigma}}, t)$ is required. This function is calculated at each integration step of (39). The $\hat{\boldsymbol{\Sigma}}_{k}$ calculated in (56) serves as the initial condition for (39) after the $k$ th data processing step.

1) The values $\tilde{\boldsymbol{x}}, \boldsymbol{Q}_{i}$ and $q_{i}$ are calculated, by minimizing the expression (45) subject to (43). When the process (1) consists of polynomials or rational functions of polynomials this step is most efficiently carried out using [4]. An example of this calculations is presented in section VI.

2) The linear program (28), subject to (21), with $\boldsymbol{\Sigma}=\tilde{\boldsymbol{\Sigma}}$ and the minimization (42) subject to (31) are solved to calculate $s$ and finally $\dot{\bar{\Sigma}}$ is obtained by substituting $s$ in (29).

\section{AN EXAMPLE}

The example in the paper is a second order system with a limit cycle,

$$
\mathrm{d} \boldsymbol{x}=\boldsymbol{A}(\boldsymbol{x}) \boldsymbol{x}+\boldsymbol{G} \mathrm{d} \boldsymbol{w}
$$

where

$$
\begin{gathered}
\boldsymbol{A}(\boldsymbol{x})=\boldsymbol{A}_{u} / m(\boldsymbol{x})+\boldsymbol{A}_{s} \\
\boldsymbol{A}_{u}=\left[\begin{array}{cc}
1 & 1 \\
-1 & 1
\end{array}\right], \boldsymbol{A}_{s}=\left[\begin{array}{cc}
-1 & 1 \\
-1 & -1
\end{array}\right] \\
m(\boldsymbol{x})=\left(1+\boldsymbol{x}^{\prime} \boldsymbol{x}\right) / 25 \\
\boldsymbol{G}=\left[\begin{array}{cc}
1 / 5 & 0 \\
0 & 1 / 5
\end{array}\right]
\end{gathered}
$$

The observation matrix is

$$
\boldsymbol{H}=\left[\begin{array}{ll}
1 & 0
\end{array}\right]
$$

and $y=\boldsymbol{H} \boldsymbol{x}+v_{k}$, is observed every $0.2 \mathrm{~s}$. The standard deviation of $v_{k}$ is 0.01 .

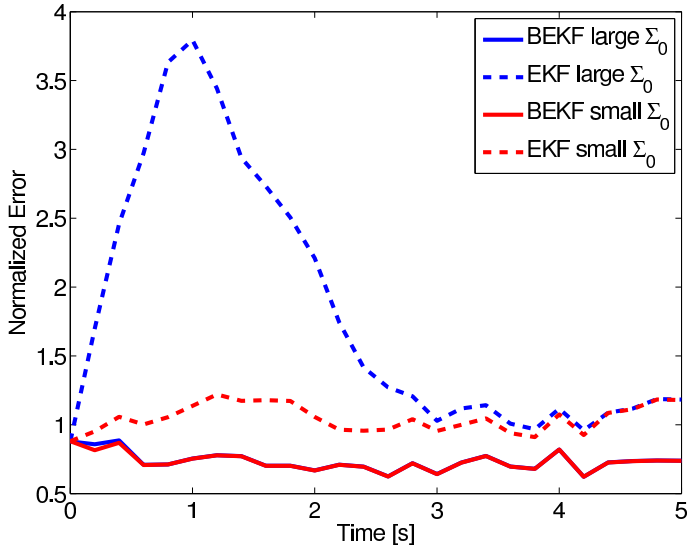

Fig. 1. Average normalized mean square error $\sqrt{\tilde{\boldsymbol{e}}^{\prime} \tilde{\boldsymbol{\Sigma}}^{-1} \tilde{\boldsymbol{e}} / 2}$

The initial step in calculating the bound is substituting into (43) yields

$$
\begin{array}{r}
{[\boldsymbol{A}(\tilde{\boldsymbol{x}}) \tilde{\boldsymbol{x}}-\boldsymbol{A}(\tilde{\boldsymbol{x}}-\tilde{\boldsymbol{e}})(\tilde{\boldsymbol{x}}-\tilde{\boldsymbol{e}})]^{\prime} \boldsymbol{P}_{i} \tilde{\boldsymbol{e}}} \\
+\tilde{\boldsymbol{e}}^{\prime} \boldsymbol{P}_{i}[\boldsymbol{A}(\tilde{\boldsymbol{x}}) \tilde{\boldsymbol{x}}-\boldsymbol{A}(\tilde{\boldsymbol{x}}-\tilde{\boldsymbol{e}})(\tilde{\boldsymbol{x}}-\tilde{\boldsymbol{e}})] \\
+\operatorname{Tr}\left\{\boldsymbol{G}^{\prime} \boldsymbol{P}_{i} \boldsymbol{G}\right\}-\tilde{\boldsymbol{e}}^{\prime} \boldsymbol{Q}_{i} \tilde{\boldsymbol{e}}-q_{i}
\end{array}
$$

This form is not suitable for use in SOSTOOLS because of the form $m(\tilde{\boldsymbol{x}}-\tilde{\boldsymbol{e}})$ in the denominator. Since $m(\tilde{\boldsymbol{x}}-\tilde{\boldsymbol{e}})>0$ multiplying (63) by $m(\tilde{\boldsymbol{x}}-\tilde{\boldsymbol{e}})$ does not affect the sign of the form, so that (63) may be replaced by

$$
\begin{array}{r}
m(\tilde{\boldsymbol{x}}-\tilde{\boldsymbol{e}})[\boldsymbol{A}(\tilde{\boldsymbol{x}}) \tilde{\boldsymbol{x}}-\boldsymbol{A}(\tilde{\boldsymbol{x}}-\tilde{\boldsymbol{e}})(\tilde{\boldsymbol{x}}-\tilde{\boldsymbol{e}})]^{\prime} \boldsymbol{P}_{i} \tilde{\boldsymbol{e}} \\
+m(\tilde{\boldsymbol{x}}-\tilde{\boldsymbol{e}}) \tilde{\boldsymbol{e}}^{\prime} \boldsymbol{P}_{i}[\boldsymbol{A}(\tilde{\boldsymbol{x}}) \tilde{\boldsymbol{x}}-\boldsymbol{A}(\tilde{\boldsymbol{x}}-\tilde{\boldsymbol{e}})(\tilde{\boldsymbol{x}}-\tilde{\boldsymbol{e}})] \\
+m(\tilde{\boldsymbol{x}}-\tilde{\boldsymbol{e}}) \operatorname{Tr}\left\{\boldsymbol{G}^{\prime} \boldsymbol{P}_{i} \boldsymbol{G}\right\}-m(\tilde{\boldsymbol{x}}-\tilde{\boldsymbol{e}})\left(\tilde{\boldsymbol{e}}^{\prime} \boldsymbol{Q}_{i} \tilde{\boldsymbol{e}}-q_{i}\right)
\end{array}
$$

Then the input to SOSTOOLS consists of the minimization of (45) subject to the constraint (64).

In Fig. 1 is shown the average normalized mean square error, $\sqrt{\tilde{\boldsymbol{e}}^{\prime} \tilde{\boldsymbol{\Sigma}}-1 \tilde{\boldsymbol{e}} / 2}$, calculated at each integration step, for the initial conditions

$$
\begin{gathered}
\boldsymbol{\Sigma}_{0}=\left[\begin{array}{cc}
0.5 & 0 \\
0 & 0.5
\end{array}\right], \boldsymbol{\mu}=\left[\begin{array}{l}
8 \\
0
\end{array}\right] \\
\boldsymbol{\Sigma}_{0}=\left[\begin{array}{cc}
0.01 & 0 \\
0 & 0.01
\end{array}\right], \boldsymbol{\mu}=\left[\begin{array}{l}
8 \\
0
\end{array}\right]
\end{gathered}
$$

Two sets of lines are shown in Fig 1: the full lines (blue and red) shows the normalized error for the BEKF filter proposed in the present paper; and the dashed lines (blue and red) the normalized error for the classical extended Kalman filter (here the error is normalized using the covariance matrix calculated by the extended Kalman filter). It is seen that for the proposed filter the normalized error is for all cases about 0.7 , implying a certain amount of conservativeness. For the extended Kalman filter, when the initial uncertainty is small red line, (66), the normalized error is near one, implying that the filter is operating well. For case (65), blue dashed line, the conventional extended Kalman filter severely underestimates the filter error, implying considerably too low a value for the filter gain. Only after about 3 seconds is does the normalized mean square error settle near 1 . Further increasing the initial 
uncertainty for the extended Kalman filter causes it to loose all connection between the calculated covariance matrix and the actual estimate error. Note that this situation may be remedied by increasing the process noise in the extended Kalman filter beyond its actual value.

\section{SUMMARY AND CONCLUSION}

A new form of the extended Kalman filter was presented. The main distinguishing feature of the new filter is the computation of a bound for the mean square error matrix for the estimate error. This matrix serves as both a bound on the actual mean square error and is used in the calculation of the Kalman gain. In contrast, the connection between the covariance matrix, computed by linearizing the dynamics about the state estimate in the conventional extended Kalman filter is at best very approximate. As a consequence, the extended Kalman has to be "tuned" by increasing the process noise amplitude, to ensure some sort of connection between the computed covariance and the actual mean square error.

The emphasis has been on deriving a mean square bound. Tighter bounds may be achievable by judicious choice of the matrices $\boldsymbol{P}_{i}$, and $\boldsymbol{U}_{i}$. Note that when the system is linear the proposed filter reduces to the conventional linear Kalman filter.

\section{APPENDIX}

\section{A. Calculating $\boldsymbol{T}_{i}$}

The purpose of this section is to calculate the $\boldsymbol{T}_{i}$ from the $\boldsymbol{U}_{i}$. As noted in section III-A.2 the bound calculated in the paper becomes tighter the better the cone generated by the set of $\boldsymbol{U}_{i}$ approximate $\mathcal{M}_{+}$, the cone of positive definite matrices. There is at present no explicit algorithm for selecting the $\boldsymbol{U}_{i}$, except for choosing the $\boldsymbol{U}_{i}$ to be rank one positive semi-definite matrices. Note that any choice of a set of positive semi-definite matrices, $\boldsymbol{U}_{i}$ results in a valid mean square error bound. but some choices for $\boldsymbol{U}_{i}$ may result in a tighter bound.

When there are $n \boldsymbol{U}_{i}$ 's, to each $\boldsymbol{U}_{i}$ there corresponds a $\boldsymbol{T}_{i}$ and is defined as follows

1) $\boldsymbol{T}_{i}$ is orthogonal to all the $\boldsymbol{U}_{k}, k \neq i$ that is $\operatorname{Tr}\left\{\boldsymbol{T}_{i} \boldsymbol{U}_{k}\right\}=0$.

2) in addition, $\operatorname{Tr}\left\{\boldsymbol{T}_{i} \boldsymbol{U}_{i}\right\}=1>0$

These two conditions define $n$ linear equations in $n$ unknowns, whose solution is straightforward. When there are more than $n \boldsymbol{U}_{i}$ 's then each subset of $n \boldsymbol{U}_{i}$ 's yields a set of $\boldsymbol{T}_{i}$ 's. From the union of all these $\boldsymbol{T}_{i}$ 's corresponding to all possible subsets of $n \boldsymbol{U}_{i}$ 's, a set of $\boldsymbol{T}_{i}$ 's is chosen as the generators of $\mathcal{F}^{*}$

1) If $\operatorname{Tr}\left\{\boldsymbol{T}_{i} \boldsymbol{U}_{j}\right\}>0$ for some $j$ then $\boldsymbol{T}_{i}$ is excluded.

2) Any $\boldsymbol{T}_{i}$ not in the set is expressible as a positive linear combination of the $\boldsymbol{T}_{i}$ 's in the set.

3) Any $\boldsymbol{T}_{i}$ in the set is not expressible as a positive linear combination of the remaining $\boldsymbol{T}_{i}$ 's in the set.

\section{B. Calculating the $\boldsymbol{P}_{i}$}

This section presents an algorithm for calculating a minimal set of $\boldsymbol{P}_{i}$ 's. The main property of the $N \times N$ symmetric matrices $\boldsymbol{P}_{i}$ is that given any $N \times N$ symmetric matrix $\boldsymbol{Z}$ there exists $i$ such that $\operatorname{Tr}\left\{\boldsymbol{P}_{i} \boldsymbol{Z}\right\}>0$. Let $\boldsymbol{P}_{1}$ be arbitrary, subject to

$$
\operatorname{Tr}\left\{\boldsymbol{P}_{1} \boldsymbol{P}_{1}\right\}=1
$$

There are $n+1 \boldsymbol{P}$ 's, and the first $n$ are defined recursively.

$$
\boldsymbol{P}_{k+1}=\alpha_{k} \sum_{i=1}^{k} \boldsymbol{P}_{i}+\beta_{k} \boldsymbol{S}_{k}, k=1,2, \ldots, n-1
$$

The $\boldsymbol{S}_{k}$ is chosen to satisfy

$$
\operatorname{Tr}\left\{\boldsymbol{P}_{i} \boldsymbol{S}_{k}\right\}=0, \text { for } i=1,2 \ldots k
$$

and

$$
\operatorname{Tr}\left\{\boldsymbol{S}_{k} \boldsymbol{S}_{k}\right\}=1
$$

Note that some of the entries of $\boldsymbol{S}_{k}$ are arbitrary, since the number of entries in $\boldsymbol{S}_{k}$ is greater than the number of equations. The constant $\alpha_{k}$ is chosen so that

$$
\operatorname{Tr}\left\{\boldsymbol{P}_{k+1} \boldsymbol{P}_{i}\right\}=-1 / n, \text { for } i=1,2, \ldots k
$$

Giving $\alpha_{k}$ the value

$$
\alpha_{k}=-\frac{1}{n-(k-1)}
$$

ensures this. The constant $\beta_{k}$ is chosen so that

$$
\operatorname{Tr}\left\{\boldsymbol{P}_{k+1} \boldsymbol{P}_{k+1}\right\}=1
$$

Choosing

$$
\beta_{k}=\sqrt{1-\frac{k}{n(n-(k-1))}}
$$

ensures this. Note that the expression under the radical is always positive. Finally the last $\boldsymbol{P}_{n+1}$ is defined

$$
\boldsymbol{P}_{n+1}=-\sum_{i=1}^{n} \boldsymbol{P}_{i}
$$

Using (73) and (71) and the definition of $\boldsymbol{P}_{n+1}$ in (75) yields

$$
\operatorname{Tr}\left\{\boldsymbol{P}_{n+1} \boldsymbol{P}_{n+1}\right\}=1
$$

A similar calculation shows that

$$
\operatorname{Tr}\left\{\boldsymbol{P}_{n+1} \boldsymbol{P}_{i}\right\}=-1 / n, i=1,2, \ldots, n
$$

From their construction the first $n \boldsymbol{P}_{i}$ span the space of $N \times$ $N$ symmetric matrices. Accordingly, if $\boldsymbol{Z}$ is any symmetric $N \times N$ matrix there exist $z_{i}$,

$$
\boldsymbol{Z}=\sum_{i=1}^{n} z_{i} \boldsymbol{P}_{i}
$$

Solving (75) for any $\boldsymbol{P}_{i}$ and substituting into (78) shows that in fact any $n \boldsymbol{P}_{i}$ 's span the space $N \times N$ symmetric matrices. Rearranging (75)

$$
\sum_{i=1}^{n+1} \boldsymbol{P}_{i}=\mathbf{0}
$$


so that

$$
\operatorname{Tr}\left\{\boldsymbol{Z} \sum_{i=1}^{n+1} \boldsymbol{P}_{i}\right\}=0
$$

Since the $\boldsymbol{P}_{i}$ 's span the space of symmetric matrices, if $\boldsymbol{Z} \neq$ $\mathbf{0}$, not all the terms in (80) are zero. Therefor there exists an $i$ such that

$$
\operatorname{Tr}\left\{\boldsymbol{Z} \boldsymbol{P}_{i}\right\}>0
$$

\section{Proof of Lemma 1}

Suppose that the Lemma is false. Then there exists $t_{1}$, and $t_{2}$ such that

$$
\boldsymbol{\Sigma}(t) \succ \tilde{\boldsymbol{\Sigma}}(t), t_{1}<t \leq t_{2}
$$

but

$$
\boldsymbol{\Sigma}\left(t_{1}\right)=\tilde{\boldsymbol{\Sigma}}\left(t_{1}\right)
$$

From the mean value theorem,

$$
\tilde{\boldsymbol{\Sigma}}\left(t_{2}\right)-\boldsymbol{\Sigma}\left(t_{2}\right)=\frac{\mathrm{d}}{\mathrm{d} t}\left(\tilde{\boldsymbol{\Sigma}}\left(t_{a}\right)-\boldsymbol{\Sigma}\left(t_{a}\right)\right)\left(t_{2}-t_{1}\right)
$$

where $t_{1} \leq t_{a} \leq t_{2}$, implying that

$$
\frac{\mathrm{d}}{\mathrm{d} t}\left(\tilde{\boldsymbol{\Sigma}}\left(t_{a}\right)-\boldsymbol{\Sigma}\left(t_{a}\right)\right) \succ \mathbf{0}
$$

which contradicts (39) and (20).

\section{REFERENCES}

[1] A. Papachristodoulou and S. Prajna, "A tutorial on sum of squares techniques for system analysis," in American Control Conference, 2005.

[2] P. A. Parrilo and S. Lall, "Semidefinite programming relaxations and algebraic optimization in control," European Journal of Control, vol. 9, no. 2-3, pp. 307-321, 2003.

[3] G. Chesi, "LMI techniques for optimization over polynomials in control: a survey," IEEE Transactions on Automatic Control, vol. AC55, no. 11, pp. 2500-2510, 2010.

[4] S. Prajna, A. Papachristodoulo, P. Seiler, and P. A. Parrilo. (2004) SOSTOOLS. [Online]. Available: http://www.cds.caltech.edu/sostools/

[5] J. F. Strum. (2010) SeDuMi. [Online]. Available: http://sedumi.ie.lehigh.edu/

[6] H. W. Sorenson, "On the development of practical nonlinear filters," Information Sciences, vol. 7, pp. 253-270, 1974.

[7] A. H. Jazwinsky, Stochastic Processes and Filtering Theory. New York: Academic Press, 1970.

[8] M. Kumar and S. Chakravorty, "Nonlinear filter based on the FokkerPlanck equation," Journal of Guidance, Control and Dynamics, vol. 35, no. 1, pp. 68-79, January-February 2012.

[9] A. S. Gilman and I. A. Rhodes, "Cone-bounded nonlinearities and mean-square bounds - quadratic regulation bounds," IEEE Transactions on Automatic Control, vol. AC-21, no. 4, pp. 472-483, August 1976.

[10] — - "Cone-bounded nonlinearities and mean square boundsestimation lower bound," IEEE Transactions on Automatic Control, vol. AC-20, no. 5, pp. 632-641, 1975.

[11] — "Cone-bounded nonlinearities and mean square boundsestimation upper bound," IEEE Transactions on Automatic Control, vol. AC-18, no. 3, pp. 260-265, 1973.

[12] Y. BarShalom, X. R. Li, and T. Kirubajan, Estimation with Applications to Tracking and Navigation: Theory Algorithm and Software. John Wiley and Sons Inc., 2001.

[13] W. Lohmiller and J. E. Slotine, "On contraction analysis for non-linear systems," Automatica, vol. 34, no. 6, pp. 683-696, 1998.

[14] Q. Pham, N. Tabareau, and J. E. Slotine, "A contraction theory approach to stochastic incremental stability," IEEE Transactions on Automatic Control, vol. AC-54, no. 4, 2009.

[15] S. Boyd and L. Vandenberghe, Convex Optimization. Cambridge University Press, 2004.

[16] H. K. Khalil, Nonlinear Systems, 3rd ed. Prentice Hall, 2002. 